\title{
ON IDEAL CLASS GROUPS OF 2-POWER EXPONENT
}

\author{
A. G. EARNEST AND O. H. KÖRER
}

\begin{abstract}
It is shown that for a fixed totally real algebraic number field $k$ and a fixed positive integer $t$, there exist only finitely many totally imaginary quadratic extensions $K$ of $k$ having ideal class groups of exponent $2^{t}$.
\end{abstract}

Under the assumption of the extended Riemann Hypothesis it has been shown by Boyd and Kisilevsky [1], and independently by Weinberger [6], that the exponent of the ideal class group of an imaginary quadratic field $\mathbf{Q}(\sqrt{d}), d$ a fundamental discriminant, tends to infinity as $|d|$ tends to infinity. It follows that for any positive integer $e$ there exist only finitely many such fields having ideal class group of exponent $e$. The particular cases $e=2,3$ and 4 of this result have been established without the Riemann Hypothesis assumption in [2, 1 and 3], respectively.

The purpose of this note is to prove an unconditional result of this nature for an infinite family of exponents in the more general context of totally imaginary quadratic extensions of an arbitrary totally real algebraic number field.

THEOREM 1. For a fixed totally real algebraic number field $k$ and a fixed positive integer $t$, there exist only finitely many totally imaginary quadratic extensions $K$ of $k$ having ideal class groups of exponent $2^{t}$.

To facilitate the discussion here, we first fix some notations. For an algebraic number field $F$ denote by $R_{F}$ the ring of algebraic integers of $F$, by $I_{F}$ the group of fractional $R_{F}$-ideals of $F$, and by $P_{F}$ the subgroup of $I_{F}$ consisting of principal ideals. $C_{F}$ will denote the ideal class group $I_{F} / P_{F}$ of $F$, whose order will be denoted by $h_{F}$, and $U_{F}$ will denote the group of units of $R_{F}$. For an ideal $J$ in $I_{F}, N(J)$ will denote the absolute norm.

Throughout the paper the fields $K$ and $k$ will be as in Theorem 1. The nontrivial $k$-automorphism of $K$ will be denoted by ${ }^{*}$, and the relative discriminant of $K$ over $k$ by $d_{K / k}$. For a fixed positive integer $t$, let

$$
G_{t}=\left\{x \in C_{K}: x^{2^{t}}=1\right\} .
$$

We will prove the following result, from which Theorem 1 follows immediately:

THEOREM 2. Let $k$ be a fixed totally real algebraic number field and $t$ a fixed positive integer. For totally imaginary quadratic extensions $K$ of $k$,

$$
\left|C_{K} / G_{t}\right| \rightarrow \infty \text { as } N\left(d_{K / k}\right) \rightarrow \infty \text {. }
$$

Received by the editors July $27,1981$.

1980 Mathematics Subject Classification. Primary 12A25, 12A50; Secondary 12A95.

Key words and phrases. Ideal class group, quadratic extension, totally real algebraic number field. 
Proof. Observe first that $C_{K} / G_{t} \cong C_{K}^{2 t}$, and

$$
\left|C_{K}^{2^{t}}\right|=\left|C_{K}\right|\left[\prod_{j=0}^{t-1}\left|C_{K}^{2^{j}} / C_{K}^{2^{j+1}}\right|\right]^{-1} \text {. }
$$

To estimate the factors in the above product it is convenient to introduce the groups

$$
H_{i}=\left\{B^{2^{i}} E^{2^{i-1}} P_{K} \in C_{K}: B \in I_{K} \text { and } E \in I_{k}\right\} \text {. }
$$

Consider the mappings $\phi_{j}: I_{K} \rightarrow C_{K}^{2^{j}} / H_{j+1}$ given by $X \mapsto X^{2^{j}} P_{K} H_{j+1}$. If $X=a Y / Y^{*}$ for $a \in \dot{K}, Y \in I_{K}$, then

$$
X^{2^{j}}=a^{2^{j}} Y^{2^{j}} /\left(Y^{*}\right)^{2^{j}}=a^{2^{j}} Y^{2^{j+1}} /(n Y)^{2^{j}},
$$

where $n Y$ is the relative norm of the ideal $Y$ in the extension $K / k$. Thus, $S$ is contained in the kernel of $\phi_{j}$, where

$$
S=\left\{a Y / Y^{*}: a \in \dot{K}, Y \in I_{K}\right\}
$$

So, for each $j$, we have

$$
\left|C_{K}^{2^{j}} / H_{j+1}\right|=\left|I_{K} / \operatorname{ker}\left(\phi_{j}\right)\right| \leqslant\left[I_{K}: S\right] .
$$

The latter index is known from the theory of quadratic extensions of algebraic number fields (see [4, §13, Satz 13]);

$$
\left[I_{K}: S\right]=h_{k}\left[N_{K / k}(K) \cap U_{k}: U_{k}^{2}\right] 2^{r-1} \text {, }
$$

where $r$ is the number of (finite) primes of $k$ which are ramified in $K$. For each $j$,

$$
\begin{aligned}
\left|C_{K}^{2^{j}} / C_{K}^{2^{j+1}}\right| & =\left|C_{K}^{2^{j}} / H_{j+1}\right|\left|H_{j+1} / C_{K}^{2^{j+1}}\right| \\
& \leqslant h_{k}\left[U_{k}: U_{k}^{2}\right] 2^{r-1} \cdot h_{k}=c_{1} 2^{r-1}
\end{aligned}
$$

for a constant $c_{1}$ depending only upon $k$. It now follows that

$$
\left|C_{K}^{2^{t}}\right| \geqslant c_{2} h_{K} /\left(2^{t}\right)^{r-1}
$$

for a constant $c_{2}$ depending upon $k$ and $t$. Applying a result of Stark [5, Theorem 2] when $k \neq \mathbf{Q}$ and the Brauer-Siegel Theorem when $k=\mathbf{Q}, h_{K}$ can be bounded from below by

$$
h_{K} \geqslant c_{3} N\left(d_{K / k}\right)^{1 / 4}
$$

with $c_{3}$ a constant depending only upon $k$. Combining the last two inequalities, we obtain

$$
\left|C_{K}^{2^{\prime}}\right| \geqslant c_{4} N\left(d_{K / k}\right)^{1 / 4}\left(2^{t}\right)^{1-r},
$$

where $c_{4}$ is a constant depending upon $k$ and $t$.

Now let $m$ be the number of (not necessarily distinct) prime factors of $N\left(d_{K / k}\right)$, and let $n=[k: Q]$. Then

$$
N\left(d_{K / k}\right)^{1 / 4}\left(2^{t}\right)^{1-r} \geqslant\left(\prod p^{1 / 4}\right)\left(2^{t}\right)^{-m n}=\prod\left(p^{1 / 4} 2^{-t n}\right)
$$


where the products are taken over the (not necessarily distinct) primes $p$ dividing $N\left(d_{K / k}\right)$. As $N\left(d_{K / k}\right)$ tends to infinity, the size of the largest prime divisor of $N\left(d_{K / k}\right)$ tends to infinity. Hence, the above product tends to infinity and the proof is complete.

\section{REFERENCES}

1. D. W. Boyd and H. Kisilevsky, On the exponent of the ideal class group of complex quadratic fields, Proc. Amer. Math. Soc. 31 (1971), 433-436.

2. S. Chowla, An extension of Heilbronn's class number theorem, Quart. J. Math. Oxford Ser. 5 (1934), 304-307.

3. A. G. Earnest and D. R. Estes, An algebraic approach to the growth of class numbers of binary quadratic lattices, Mathematika 28 (1981), 160-168.

4. H. Hasse, Bericht über neuere Untersuchungen und Probleme aus der Theorie der algebraischen Zahlkörpern, Teil I, Ia, 2 Aufl., Würzburg-Wien, 1965.

5. H. M. Stark, Some effective cases of the Brauer-Siegel Theorem, Invent. Math. 23 (1974), 135-152.

6. P. Weinberger, Exponents of the class groups of complex quadratic fields, Acta Arith. 22 (1973), 117-124.

Department of Mathematics, SOUThern Illinois University, Carbondale, Illinois 62901

Abteilung fúr Mathematik IV, Universitat Ulm, Oberer Eselsberg, 79 Ulm, BR Deutschland 